\title{
Solar Telescope at Oxford
}

$\mathrm{T}$ HE new solar telescope of the University Observatory, Oxford, which was formally declared open by the Vice-Chancellor on June 11, is in its essence a vertical Cassegrain telescope of $12 \frac{1}{2}$ inches aperture and 73 feet focal length. It gives a solar image some eight inches in diameter, and unlike the original tower telescopes (the $60-\mathrm{ft}$. and the $150-\mathrm{ft}$. at Mount Wilson) the present instrument is wholly free from chromatic aberration. Further, since the image is formed on the optical axis, the telescope is free from the extra axial aberrations characteristic of horizontal solar reflecting telescopes. Light is fed into the vertical Cassegrain telescope by a 16-in. colostat, driven by a synchronous motor, and a secondary mirror of the same aperture. Both cœlostat and secondary mirror are provided with electrical slow motions which are controlled by a portable keyboard near the focal plane of the instrument; the same keyboard also carries controls for focusing the Cassegrain mirror.

The telescope is unique in that it is the only large solar telescope in which all the optical parts are made of fused silica. Since fused silica has a coefficient of expansion 1/20 that of ordinary glass, and $1 / 7$ that of pyrex, it follows that the differential expansion produced by solar radiation, which tends to make the plane mirrors concave and so to produce an astigmatic image, will be almost wholly avoided in this instrument. The telescope, mounted in the east tower of the observatory on the same pier and under the same dome which housed the De la Rue reflector, was constructed by Sir Howard Grubb, Parsons and Co., of Newcastle, the blanks for the fused silica discs having been supplied by the Thermal Syndicate, Ltd.

On the optical axis of the telescope lies the slit of a large Littrow spectrograph. The spectrograph has two $60^{\circ}$ and one $30^{\circ}$ prisms of six inches square aperture (height 6 inches, length of face $9 \cdot 7$ inches), and a collimating-camera lens of six inches aperture and some 30 feet focal length. It gives a dispersion at $\lambda 4200$ of $2.6 \mathrm{~mm}$. per A., and a theoretical resolving power of more than 300,000 . The optical parts, supplied by Adam Hilger, Ltd., and a large minimum deviation mount, supplied by C. F. Casella and Co., Ltd., are mounted at one end of a 40 -ft. brick tunnel constructed in the basement of the observatory. The spectrograph, which still lacks the essential temperature control for the prisms, the slit end and plate holder mechanism, and a guiding dise, will be completed as funds permit.
In declaring this equipment open, the Vice. Chancellor referred to the chequered history of attempts to establish a permanent observatory in Oxford. No less than three unsuccessful attempts have been made, namely Bishop Fell's failure to persuade Wren to incorporate an observatory in Tom Tower, the establishment of a temporary observatory for Halley in 1704 in New College Lane, and finally the construction of an observatory by the Radcliffe Trustees in 1773, now regrettably, from the point of view of Oxford at least, departing for South Africa. The University Observatory was built by the University some sixty years ago, and the fact that the University has, during a period of exceptional financial stringency, set aside some $£ 3,700$ for the purchase of the present equipment may be regarded as evidence both that it desires the present observatory to be a permanent institution, and that it desires to see the study of observational astronomy actively prosecuted at Oxford. The Vice-Chancellor concluded his remarks by expressing the warm hope that some donor, interested in the study of astronomy, would find it possible to provide the relatively small sum needed to complete this equipment.

After the Vice-Chancellor's declaration, and following a period when the instruments were inspected by the assembled company of astronomers, Oxford men of science and other members of the University, the proceedings were concluded by an address by Sir Arthur Eddington on "The Physics of the Sun". Sir Arthur pointed out that the sun may be regarded as composed of three parts, an airy appendage where the atoms are supported by radiation pressure, a middle region where the absorption lines are formed and which is therefore readily accessible for observation with the equipment which had just been inspected, and finally the observationally inaccessible deep-lying interior. While there is a temptation to separate what is observable from what can only be inferred, the creation of such artificial divisions is likely to lead to 'frontier incidents'. The sun is a unit and must be treated as such. Sir Arthur then went on to point out how, in spite of its inaccessibility, it is possible to infer much about the physical conditions in the deep interior, and how such information has been confirmed in a striking way by developments in nuclear physics. In moving a vote of thanks to the lecturer, Prof. E. A. Milne, in a peculiarly happy vein, referred to the many distinguished astronomers and solar physicists who gathered to witness the ceremony and to hear Sir Arthur's masterlv address.

H. H. P.

\section{Interpretation of Spectra}

$\mathrm{P}$ ROF. HENRY NORRIS RUSSELL delivered the George Darwin Lecture to the Royal Astronomical Society at the meeting of the Society on June 14, taking as his subject "The Analysis of Spectra and its Application to Astronomy". Prof. Russell explained that he did not refer to the analysis of spectra in the sense of the analysis of the elements present or absent in a mixture, but to the analysis of the spectrum of each single element.
The history of this analysis goes back to 1883 , when it was discovered that the various doublets in the spectrum of sodium possessed the same separation when expressed in wave numbers: Prof. Russell followed the development of this subject down to its rationalisation by Bohr and the introduction of the notion of states of energy which the atom may possess, the frequencies of the spectral lines being given by the familiar quantum relation $h \nu=E_{1}-E_{2}$. 